\title{
MODIFIED SOBEL MASK TO LOCATE KNEE JOINT BOUNDARIES
}

S. Sheik Abdullah

Research Scholar, Department of Electronics and Communication Engineering,

Kalasalingam Academy of Research and Education, Virudhunagar, (India). E-mail: sabdullah787@gmail.com ORCID: https://orcid.org/0000-0001-6765-8374

M. Pallikonda Rajasekaran

Professor, Department of Electronics and Communication Engineering, Kalasalingam Academy of Research and Education. Virudhunagar, (India). E-mail: mpraja80@gmail.com ORCID: https://orcid.org/0000-0001-6942-4512

\section{Citación sugerida:}

Abdullah, S. S., y Rajasekaran, M. P. (2020). Modified sobel mask to locate knee joint boundaries. 3C Tecnología. Glosas de innovación aplicadas a la pyme. Edición Especial, Marzo 2020, 195-205. http://doi. org/10.17993/3ctecno.2020.specialissue4.195-205

\section{Suggested citation:}

Abdullah, S. S., \& Rajasekaran, M. P. (2020). Modified sobel mask to locate knee joint boundaries. 3C Tecnología. Glosas de innovación aplicadas a la pyme. Edición Especial, Marzo 2020, 195-205. http://doi. org/10.17993/3ctecno.2020.specialissue4.195-205 


\section{ABSTRACT}

Sobel masking algorithm is a very important technique to detect edges in an image. Comparing the Sobel gradient operator with other edge/boundary detection operators used repeatedly; Making an additional study on the traditional Sobel gradient operator, the benefits of Sobel mask are its quick speed of detecting edges. Meanwhile, it has also an impact on suppressing and smoothing noise. In addition, Sobel operator has a standard effect on detecting the edges. Although Sobel gradient operator has some advantages in different aspects, it exists some problems. The Existing Sobel masking technique is a type of edge detection in vertical and horizontal directions only and it ignores the boundary points in other directions. It cannot attain a true location of edge points in an image. In this paper, the existing sobel technique is improved by adding an increase of 315 degrees and 360 degrees in horizontal and vertical directions. This will have an effect of detecting the knee joint space of osteoarthritis. According to simulation results, they show this method is very simple and feasible, and the outcomes are more abundant than traditional Sobel edge detection. In this paper edge detection and noise interference problems are improved.

\section{KEYWORDS}

Osteoarthritis, Sobel mask, Image Processing Techniques. 


\section{INTRODUCTION}

In digital image processing, edge/boundary feature is one of the very important characteristics of the image, and it is a significant part of image processing, analyzing, pattern recognition and computer vision. Edge detection outcomes affect further image analyzing and pattern/texture recognition directly (Amer \& Abushaala, 2015). In recent days, Image edge detection has become the main research theme in image processing technology. With the advance of science and technology, researchers have analyzed and proposed some techniques for the detection of edges in an image and assessment of edge detection. At the same time, these edge/boundary recognition methods are applied to the area of digital vision and pattern recognition, which make the use of edge detection technology more broadly. Over the years, segmentation of an image has been creating more and more attention. Lots of image segmentation techniques have been put forward. They can be divided into different methods like bit threshold method, edge detection method and regional growth method (Argyle, 1971; Canny, 1989). Edge detection method comprises of: edge detection operator which contains mask like Roberts operator, Prewitt operator, LOG operator and Sobel operator (Abbasi \& Abbasi, 2007). Sobel mask is slightly better than others. The classical Sobel technique also has some problems such as it is sensitive to the vertical and horizontal direction only (Lakshmi \& Sankaranarayanan, 2010). However, the information in the image is not restricted to the horizontal and vertical directions; it can make an element of the image information lose. In this paper, a new improved operator is proposed to detect more image information. In the modified Sobel operator, 2 direction patterns (315 degrees and 360 degrees) are added to get multi-directional image acquisition. Then calculate the threshold by using the Otsu method and refine the detected rough edges by using the method to achieve the results of image edge detection. Edge detection effect can be achieved better by using the Matlab simulation method.

\section{LITERATURE SURVEY-COMPARISION OF TRADITIONAL EDGE DETECTION OPERATORS}

Roberts operator: It did not pass smooth analysis, so it is very sensitive to the noise.

Prewitt operator and Sobel operator: extraction of edge/boundaries effect is almost the same (Lakshmi \& Sankaranarayanan, 2010; Abbasi \& Abbasi, 2007). Sobel operator is a weighted average filter, Prewitt operator is an average filter; Sobel operator have better 
detection effect on images which have low level noise, but the detection of the edge effect is not clear.

LOG operator: detecting edges by using second order derivatives zero crossing edge method (Yu-quian, Wei-hua, Zhen-cheng, Jing-tian, \& Ling-yun, 2005). Smoothing effect is more important, noise removal is improved, but the loss of information in an image is higher, the edge accuracy is lower. So there is a challenge between placing edge accuracy and removing noise level.

\section{TRADITIONAL SOBEL OPERATOR}

Sobel operator, because of its task in the pattern is small, the computation is also very small, and the image information of the shape can be attained. Operator template size is even, the pending pixel cannot be placed in the center position of the template. Sobel differential mask is a differential mask of $3 \times 3$ size template (Argyle, 1971). The expressions of formula as follow:

$$
\begin{array}{r}
G x(u, v)=f[u-1, v+1]+2 * f[u, v+1]+f[u+1, v+1]- \\
f[u-1, v-1]-2 * f[u, v-1]-f[u+1, v-1] \\
f[u-1, v-1]-2 * f[u, v-1]-f[u+1, v-1] \\
G x(u, v)=f[u-1, v-1]+2 * f[u+1, v]+f[u+1, v+1]- \\
f[u-1, v-1]-2 * f[u-1, v]-f[u-1, v+1] \\
f[u-1, v-1]-2 * f[u-1, v]-f[u-1, v+1]
\end{array}
$$

The convolution template of the Sobel operator is expressed as the formula

$$
G x=\left[\begin{array}{rrr}
-1 & -2 & -1 \\
0 & 0 & 0 \\
1 & 2 & 1
\end{array}\right] \quad G y=\left[\begin{array}{rrr}
-1 & 0 & 1 \\
-2 & 0 & 2 \\
-1 & 0 & 1
\end{array}\right]
$$

The calculating steps of Sobel operator: first, the edge detection image is divided into matrix form 


$$
\left[\begin{array}{ccc}
f(x, y) & f(x+1, y) & f(x+2, y) \\
f(x, y+1) & f(x+1, y+1) & f(x+2, y+1) \\
f(x, y+2) & f(x+1, y+2) & f(x+2, y+2)
\end{array}\right]
$$

Multiply the vertical direction by horizontal direction of the template,

$$
\begin{aligned}
& F x=G x . * A \\
& F x=G y \cdot * A
\end{aligned}
$$

gradient size calculation, as shown in the formula

$$
G=\sqrt{G x^{2}+G y^{2}}
$$

The formula for calculating the gradient direction is shown in the formula

$$
\theta=\tan ^{-1}(G y / G x)
$$

The Sobel mask set up the weighted local average, The operator not only influences the edge detection of an image but also hold back the noise further, but the edge is wider. The basic idea of Sobel operator algorithm: The edge of the image is situated at the place in which the brightness varies significantly (Kalpana \& Padmaa, 2014), the gray value of pixels exceeds a set threshold depending on the specific steps for the edge (Xing, 2005). The specific steps of the Sobel operator algorithm are as follows:

- Moving the horizontal and vertical direction templates from right to left, from top to bottom, and moving from one pixel to another.

- Multiplying the pixel values in the image with operator coefficient.

- Calculated gradient value is the new gray value by using 2 convolution values.

\section{IMPROVED SOBEL OPERATOR}

Adding 315 degrees and 360 degrees with respect to the template in a basis of the traditional Sobel operator, the direction template is changed into two directions ( $G x=315$ degree, $G y=360$ degree): the horizontal and vertical direction with respect to 315 degrees and 360 degrees. It 
improved the weights of the new template in the direction of boundaries. Specific details are as follows:

According to the calculation of the two template directions and calculating an image point by point, the maximum value is observed as the pixel gray values. According to the threshold setting, the edge point is determined.

$$
\begin{gathered}
S x(o, p)=f[o+2, p+1]+2 * f[o+2, p+2]+f[o+1, p+2]- \\
f[o+1, p]-2 * f[o, p]-f[o, p+1] \\
f[o+1, p]-2 * f[o, p]-f[o, p+1] \\
S x(o, p)=f[o, p+2]+2 * f[o+1, p+2]+f[o+2, p+2]- \\
f[o, p]-2 * f[o+1, p+2]-f[o+2, p] \\
f[o, p]-2 * f[o+1, p+2]-f[o+2, p]
\end{gathered}
$$

Start

$S x=\left[\begin{array}{rrr}-3 & -1 & 0 \\ -1 & 0 & 0 \\ 0 & 1 & 2\end{array}\right] \quad S y=\left[\begin{array}{ccc}-1 & -2 & -1 \\ 0 & 0 & 0 \\ 1 & 2 & 1\end{array}\right]$

\section{Convert RGB image to grayscale}

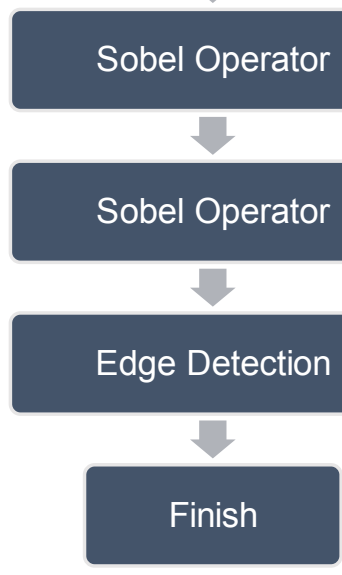

Figure 1. Flowchart of proposed system. 


\section{RESULTS}

Edge detection process followed below:

Step 1: Set Threshold value $\mathrm{T}=255$.

Step 2: If Gradient value $(\mathbf{S})<255$ is less than the Thresh, considered as 1 , other than are 0 (value below 255 set to be 0 ).

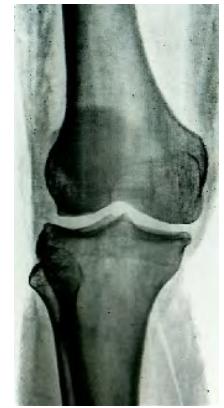

Figure 2. Original Image.

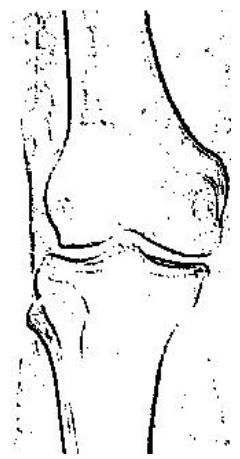

Figure 4. Sobel Operator.

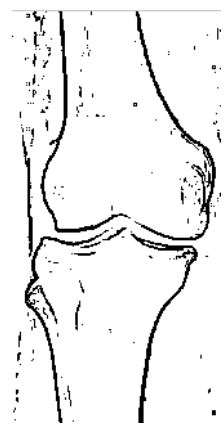

Figure 6. Prewitt Operator.

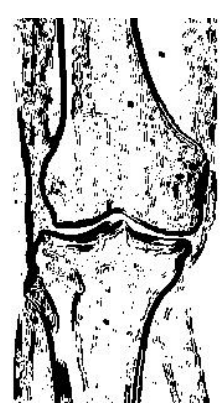

Figure 3. Proposed System.

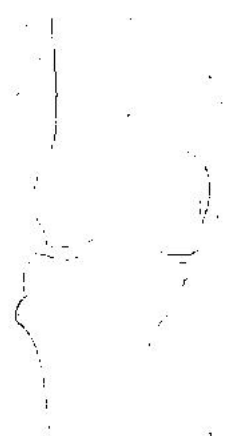

Figure 5. Roberts Operator.

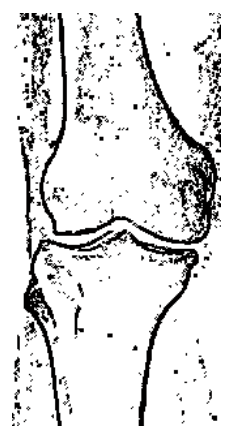

Figure 7. Homogeneity Operator. 
Table 1. PSNR comparison of different operators.

\begin{tabular}{|c|c|c|c|c|c|c|}
\hline $\begin{array}{c}\text { Test } \\
\text { images }\end{array}$ & Sobel & Zero Cross & Prewitt & Roberts & Homogeneity & $\begin{array}{c}\text { Modified } \\
\text { Sobel }\end{array}$ \\
\hline 01 & +19.39 & +13.48 & +18.51 & +14.25 & +10.23 & +20.95 \\
\hline 02 & +19.58 & +13.92 & +18.76 & +14.73 & +10.18 & +21.34 \\
\hline 03 & +19.63 & +13.79 & +13.71 & +14.63 & +10.27 & +21.15 \\
\hline 04 & +19.68 & +13.86 & +18.80 & +15.21 & +10.20 & +21.22 \\
\hline 05 & +20.03 & +13.76 & +18.96 & +14.32 & +10.31 & +21.49 \\
\hline 06 & +19.99 & +13.84 & +19.22 & +16.23 & +10.22 & +21.30 \\
\hline 08 & +19.88 & +13.86 & +19.00 & +16.10 & +10.19 & +21.45 \\
\hline 10 & +19.43 & +13.87 & +18.63 & +15.34 & +10.21 & +21.03 \\
\hline 11 & +19.63 & +13.67 & +18.72 & +15.64 & +10.41 & +21.21 \\
\hline 09 & +19.13 & +13.82 & +18.16 & +13.62 & +10.23 & +20.86 \\
\hline
\end{tabular}

\section{CONCLUSION}

This paper analyzes the classic sobel edge detection algorithm and improves the algorithm from the gradient calculation. The improved algorithm is realized that result outcomes prove that the modified algorithm is better and clearer on the edge detection of the image. From experiment, it proved that this proposed system is better than the traditional Sobel operator in image edge detection and achieves the specific accurate detection and reduces the loss of edge. The experiments show that the method provided in this paper is feasible. Improve the masking performance by increasing PSNR value and detect finite boundaries/ edges of intra articular space in future. 


\section{REFERENCES}

Abbasi, T. A., \& Abbasi, M. U. (2007). A novel FPGA-based architecture for Sobel edge detection operator. International Fournal of Electronics, 13(9), 889-896. https://doi. org/10.1080/00207210701685253

Amer, G. M. H., \& Abushaala, A. M. (2015). Edge Detection Methods. In G. Deng, Z. Liu. Comparison and Analysis for Edge Detection Algorithms based on SQI Image Enhancement. IEEE, 3615-3617.

Argyle, E. (1971). Techniques for edge detection. IEEE proceedings, 59, 285-286.

Ganny, J. (1989). A computational approach to edge detection. IEEE Transactions on Pattern Analysis and Machine Intelligence, PAMI-8(6), 679-698. https://doi.org/10.1109/ TPAMI.1986.4767851

Kalpana, Y. B., \& Padmaa, M. V. (2014). An efficient edge detection algorithm for flame and fire image processing. In 2014 International Conference on Communication and Signal Processing, 696-700. https://doi.org/10.1109/ICGSP.2014.6949932

Lakshmi, S., \& Sankaranarayanan, V. (2010). A study of Edge Detection Techniques for Segmentation Computing Approaches. IfCA Special Issue on Computer Aided Soft Computing Techniques for Imaging and Biomedical Applications. https:/ / pdfs.semanticscholar. org/3c0e/8119096edd337002d8430c6c6ad69f126520.pdf

Rama Bai, M. (2010). A new approach for border extraction using morphological methods. International Fournal of Engineering Science and Technology, 2, 3832-3837.

Xing,J. (2005). Digital image edge detection based on Sobel operator. Fournal of microcomputer development, 18-19.

Yu-quian, Z., Wei-hua, G., Zhen-cheng, G., Jing-tian, T., \& Ling-yun, L. (2005). Medical Images Edge detection Based on mathematical Morphology. In 2005 IEEE Engineering in Medicine and Biology 27th Annual Conference, Shanghai, China. https://doi. org/10.1109/IEMBS.2005.1615986 
Zhang, R., Zhao, G., \& Su, L. (2005). A New Edge Detection Method in Image Processing. IEEE International Symposium on Communications and Information Technology. ISCIT, 1, 445448. https://doi.org/10.1 109/ISCIT.2005.1566889 
